\title{
Corrigendum
}

\section{Heritability and whole genome linkage of pulse pressure in Chinese twin pairs - Corrigendum}

\author{
Wengjie Jiang, Dongfeng Zhang, Zengchang Pang, Shuxia Li, Haiping Duan, Shaojie Wang \\ and Qihua Tan \\ doi:10.1017/thg.2012.58, Published by Cambridge University Press 2012.
}

The author Wengjie Jiang name has been spelt incorrectly, it should appear as:

Wenjie Jiang

\section{Reference}

Wengjie Jiang, Dongfeng Zhang, Zengchang Pang, Shuxia Li, Haiping Duan, Shaojie Wang, and Qihua Tan. (2012). Heritability and whole genome linkage of pulse pressure in Chinese twin pairs. Twin Research and Human Genetics. 